\title{
Método de contornos ativos pSnakes aplicado em imagens de ecocardiograma de eixo curto para cálculo de fração de ejeção
}

\author{
Auzuir Ripardo de Alexandria*, Paulo César Cortez, John Hebert da Silva Felix, José Sebastião de Abreu
}

\begin{abstract}
Resumo Este trabalho propõe um método de contornos ativos, denominado pSnakes e o avalia no cálculo da fração de ejeção do ventrículo esquerdo em imagens de ecocardiograma de eixo curto. O pSnakes usa coordenadas polares para representar os pixels da imagem na modalidade ultrassom, cuja origem dos eixos de referência é localizada no ponto de divergência dos feixes ultrassônicos e usa a transformada de Hilbert para calcular a energia externa no pSnakes, denominada de energia hilbertiana. A fração de ejeção (FEJ) de cada paciente é calculada e os resultados são comparados com os resultados obtidos por dois médicos especialistas (ECO1 e ECO2). A fração de ejeção é novamente calculada utilizando-se os métodos de contornos ativos tradicional (ST), snakes radial com derivada (SRD), snakes radial hilbertiano (SRH) e pSnakes, para inicializações diferentes: na borda, $30 \%, 50 \%$ e $70 \%$ mais distante das bordas. Consegue-se um desvio da medida da fração de ejeção do ventrículo esquerdo empregando-se o método pSnakes de $10 \pm 7 \%$ em relação à medição manual do ecocardiografista ECO1 e $10 \pm 9 \%$, em relação a ECO2. O pSnakes obteve a maior correlação entre ECO1 e os métodos avaliados em todas as situações analisadas. Assim, pode-se concluir que o método pSnakes pode ser aplicado na segmentação de imagens de ecocardiograma visando o cálculo de FEJ, além da utilização da transformada de Hilbert como energia externa no método pSnakes.
\end{abstract}

Palavras-chave Contornos ativos, Snakes radiais, Transformada de Hilbert, Energia hilbertiana, pSnakes, Segmentação de imagens, Ecocardiograma.

\section{pSnakes active contours method applied on short axes echocardiogram images to ejection fraction calculation}

\begin{abstract}
This study proposes a radial active contours method named pSnakes, and applies it to the calculation of the ejection fraction of left ventricle in short axes echocardiogram images. The pSnakes uses polar coordinates to represent the image pixels in the ultrasound mode. The origin of reference axes is located in the divergence beam point from the ultrasound probe. The Hilbert transform is used in this method to calculate the external energy in pSnakes being denominated as Hilbertian energy. We calculated the ejection fraction of each patient exam and compared the results to the ones obtained by two experts (ECO1 and ECO2). Then the ejection fraction is recalculated using the traditional active contours methods (ST), snakes radial with derivative (SRD), Hilbertian radial snakes (SRH) and pSnakes to different initializations: on the edge and 30\%, 50\%, 70\% farther from the border. We obtained an error of ejection fraction of left ventricle using pSnakes method of $10 \pm 7 \%$ for ECO1 and $10 \pm 9 \%$ regarding to ECO2. For the correlation between the evaluated methods and ECO1, pSnakes scored best in all situations. We can conclude that the pSnakes method can be applied in ejection fraction calculation and segmentation of images of left ventricle in echocardiogram images, and that the Hilbertian can be used as pSnakes external energy.
\end{abstract}

Keywords Active contours, Radial snakes, Hilbert transform, Hilbertian energy, pSnakes, Image segmentation, Echocardiogram. 


\section{Extended Abstract}

\section{Introduction}

Data obtained from the left ventricle in echocardiogram images are important information taken into consideration in medical diagnosis of myocardial (Costa Filho et al., 1999). To assist in the diagnosis and reducing the subjectivity of the results, Computer Vision techniques, such as segmentation, were developed (Caiani et al., 2001). Among these techniques, we highlight the active contours also known as snakes (Kass et al., 1987). These techniques consist of building splines as the limits of the inner cavity of the left ventricle are being identified.

These contours are still being shaped as the left ventricle inner walls, in accordance with the movement of these walls. The contour deformation is performed by finding the curve of lower total energy; this energy depends on the snake geometry (internal energy) and the characteristic of the image (external energy). The snake's external energy is traditionally calculated by gradient (Kass et al., 1987).

Among the active contours methods, we have the methods of radial active contours (Denzler e Niemann, 1999). These were developed in order to reduce the complexity of active contours computational methods and making possible the application in situations of real time. The energy calculations and minimization are performed in one dimension (1D).

The method of active contours pSnakes uses the polar coordinates to represent the pixels from the image in the ultrasound mode (Alexandria et al., 2009). The axis reference origin is located at the point of the ultrasound beam divergence, i.e., the point where it finds the ultrasonic probe.

This paper proposes a method of active contours called pSnakes and applies it to the calculation of the ejection fraction of left ventricle in short axes echocardiogram images.

\section{Materials and Methods}

The method is implemented and the simulations are processed in the MATLAB ${ }^{\circledR}$ environment, version 7.6, R2008a. Both synthetic and real images are analyzed. Hilbertian energy is used as external energy for pSnakes. To minimize the total energy from the radial snake we use the greedy algorithm with search space through the ray.

pSnakes method was applied to short long axes echocardiogram images. These images were obtained from ultrasonic equipment (GE Vivid 7 PRO) from Clínica Prontocárdio in Fortaleza, Ceará and were manually segmented by two expert physicians to be compared to $p$ Snakes segmentation. Through the segmentation of the left ventricle in echocardiogram images, we calculated the ejection fraction of each patient exam and we compared the obtained results to the ones obtained by two experts (ECOI and ECO2). Then the ejection fraction is recalculated using the traditional active contours methods (ST), snakes radial with derivative (SRD), Hilbertian radial snakes (SRH) and pSnakes to different initializations: on the edge and 30\%, 50\%, 70\% farther from the border. The mean and standard deviation of the ejection fraction are obtained; and also the correlation for ECO1, since he is the most experienced of the two echocardiographers.

\section{Results and Discussion}

The method is applied to 17 short axes echocardiogram exams with manual initialization. We calculated the ejection fraction of each patient exam and we compared the obtained results to the ones obtained by two experts. We obtained, in average, an error of ejection fraction of left ventricle using $p$ Snakes method of $10 \%$ and $10 \%$, with standard deviation of $7 \%$ and $9 \%$, respectively regarding to manual measurements of each echocardiogram expert. The interobservator error between the measurements of ECO1 and ECO2 is $9 \pm 6 \%$ of standard deviation.

Comparing the calculation of ejection fraction obtained by pSnakes and the results obtained by ST, SRD and SRH, it is observed that the pSnakes had an adequate average with the best standard deviation (71.8 \pm 10.9$)$, compared to ECO1. SRH had the best average, but the standard deviation obtained was very high (70.8 \pm 14.7$)$. ST with $71.8 \pm 12.6$ was also nearby. Only SRD was far away from the average ECO1, and with lower standard deviation $(79.0 \pm 8.9)$. Regarding to the correlation between the evaluated ACM and ECO1, pSnakes scored best in all situations, obtaining 0.83 (50\% far from the borders) distantly followed by SRD and ST with 0.50 (50\% far) and 0.48 (70\% far), respectively. SRH reached the lowest correlation results (below 0.20). Regarding to processing time for each image, SRD SRH takes on average $0.56 \mathrm{~s}$ in each image of echocardiography, whereas pSnakes takes $0.69 \mathrm{~s}$. ST takes $1.79 \mathrm{~s}$.

\section{Conclusions}

The pSnakes method is tested using synthetic and echocardiogram images, being the obtained results very efficient for the tested images. Then, we can conclude from the obtained results in synthetic and real images that the pSnakes method can be applied in ejection fraction calculation and segmentation of images of left ventricle in echocardiogram images. We also conclude that the Hilbertian energy calculated through the Hilbert transform application along the radial beam can be used as pSnakes external energy. 


\section{Introdução}

O desenvolvimento de sistemas computacionais em sintonia com o conhecimento médico tem possibilitado a construção de equipamentos com importantes contribuições na identificação de doenças e seus tratamentos. $\mathrm{O}$ uso desses equipamentos está largamente presente na medicina, desde simples exames laboratoriais até exames de elevada complexidade (Webster, 1998).

Equipamentos como os tomógrafos e aparelhos de ultrassom têm possibilitado importantes avanços em diagnósticos e seu uso está disseminado na medicina e telemedicina. Empresas desenvolvem equipamentos voltados a proporcionar maior agilidade e precisão nos diagnósticos médicos. Como principais características destes equipamentos, podem ser mencionadas: produtividade e menor tempo de diagnósticos; detalhes em imagens inalcançáveis à percepção natural da visão humana; maior confiabilidade nas análises realizadas e capacidade de proporcionar aos cirurgiões maior segurança quanto aos procedimentos (Markman Filho, 2006).

Dentre os equipamentos desenvolvidos para o auxílio ao diagnóstico, encontram-se: eletroencefalógrafo, eletrocardiógrafo, ecocardiógrafo, angiógrafos, endoscópicos. Tais equipamentos são utilizados em diversas áreas da medicina, mas todos têm em comum as imagens e/ou gráficos como representação do estado do objeto em análise.

$\mathrm{O}$ aparelho de ecocardiograma produz imagens das estruturas internas do músculo cardíaco através da emissão de ondas de som que, ao incidirem no mesmo, se propagam ou são refletidos em ângulos que dependem diretamente das características físicas dos tecidos. As ondas, ao retornarem (eco) ao transdutor, são processadas e representadas graficamente na forma de imagens que são apresentadas em monitores de vídeo ou impressas. A partir desta imagem o especialista realiza as análises e considerações a respeito das condições de saúde de seus pacientes.

Uma importante informação que é considerada no diagnóstico médico do miocárdio são os dados obtidos do ventrículo esquerdo. Por meio das análises desta cavidade, o especialista pode então proceder ao seu diagnóstico (Costa Filho et al., 1999). O cálculo de volume, capacidade de deformidade e área do ventrículo esquerdo são exemplos de informações importantes para a detecção de isquemia cardíaca, bem como para o planejamento cirúrgico.

Para ajudar no diagnóstico, diminuindo a subjetividade dos resultados, técnicas de visão computacional, como a segmentação, foram desenvolvidas (Caiani et al., 2001). Dentre estas destacam-se os contornos ativos, também conhecidos como snakes (Ebadollahi et al., 2002; Kass et al., 1987). Tais técnicas consistem em construir segmentos de retas (ou splines) até os limites da área interna da região a ser segmentada, no caso de inicialização dentro do objeto. Esses contornos continuam sendo moldados conforme a forma da região a ser segmentada, de acordo com os deslocamentos ocorridos nos referidos contornos. Dessa forma, os pontos de controle que orientam os segmentos de retas ou as aproximações das curvas geradas (dependendo da técnica adotada) estão em constante atualização. O comportamento dos segmentos de reta é semelhante a uma cobra, daí o nome snake.

A deformação do contorno é realizada pela busca da curva de menor energia total, energia esta que depende da geometria do snake, caracterizada como energia interna, e das características da imagem que correspondem à energia externa. A energia externa de um snake é tradicionalmente calculada em função do cálculo do gradiente (Kass et al., 1987).

Contornos ativos pertencem, na realidade, a um conjunto de algoritmos conhecidos como modelos deformáveis, com inúmeras aplicações na segmentação de imagens médicas (Bouhours, 2006; Silva et al., 2004). Outros elementos deste conjunto podem ser citados, como: Level set ou snakes geométrico (Hodneland et al., 2009; Osher e Paragios, 2003); Active Shape Models (ASM) (Cootes et al., 1999); deformable templates (Nixon e Aguado, 2008); modelos (deformáveis) probabilísticos (Staib e Duncan, 1992) e modelos probabilísticos com modelagem da forma global (Staib e Duncan, 1992; Terzopoulos e Metaxas, 1991).

Dentre os métodos de contornos ativos, destacamse os métodos de contornos ativos radiais, que foram desenvolvidos com o intuito de diminuir a complexidade computacional dos métodos de contornos ativos, podendo ser aplicados em situações de tempo real (Buda et al., 1983). Os cálculos de energia e sua minimização são realizados em uma dimensão (1D), tornando-os mais rápidos que seus similares em duas dimensões (2D) (Denzler e Niemann, 1999).

Em todos os métodos de contornos ativos é necessária a obtenção de pontos de controle (nós) presentes, no caso de imagens, nas bordas dos objetos a serem segmentados, ou seja, em regiões de altas frequências e energia. Assim, por possuir características peculiares na detecção de bordas em sinais e por ser obtida a partir da transformada de Fourier (TF), a transformada de Hilbert (TH) é utilizada neste trabalho para compor a energia hilbertiana, usada como energia externa em métodos de contornos ativos (Sarkar et al., 2009). 
O método de contornos ativos pSnakes utiliza coordenadas polares para a representação dos pixels de imagem na modalidade ultrassom (Alexandria et al., 2009). Na realidade, esse é o sistema de coordenadas mais natural para se trabalhar com ultrassonografia, visto que é desta forma que as medidas e, portanto, as imagens são obtidas. A origem dos eixos de referência no pSnakes é localizada no ponto de divergência dos feixes de ultrassom, ou seja, no ponto onde se localiza a sonda ultrassônica. Nos atuais equipamentos médicos, as imagens são geradas em coordenadas polares para, em seguida, serem convertidas para as coordenadas cartesianas, adequando-se à visualização nos monitores destes equipamentos.

O método pSnakes foi registrado no depósito de patente Alexandria et al. (2009), porém sem apresentação de avaliações do método e sem aplicação no cálculo de fração de ejeção através de imagens de ecocardiograma. A energia hilbertiana também foi apresentada na referida patente, assim como em Alexandria et al. (2010), porém sem aplicação na segmentação de imagens de ecocardiograma e sem cálculo de fração de ejeção, até este momento.

Com base na discussão anterior, este trabalho propõe um método de contornos ativos denominado pSnakes e o avalia no cálculo de fração de ejeção do ventrículo esquerdo em imagens de ecocardiograma.

\section{Métodos}

Os contornos ativos (snakes) permitem segmentar as imagens por detecção de contornos (Kass et al., 1987). Este método é aplicado com sucesso em vários problemas de visão computacional, tais como a detecção de bordas e rastreamento de objetos, entre outros. Ainda não é possível uma única solução para todas as aplicações, por causa da originalidade de cada problema caracterizado por imagens específicas em cada aplicação. Entretanto, os snakes inovaram na solução de problemas em que a detecção de bordas por gradiente não obtém sucesso, devido aos contornos com pouco contraste, a presença de ruído, entre outras causas. Os métodos de contornos ativos consistem em traçar uma curva inicial em torno ou dentro de um objeto de interesse. Esta curva se deforma, segundo algumas forças que a deslocam até encontrar as bordas do objeto. Assim, a curva é obtida por meio de iterações sucessivas de minimização de uma energia previamente especificada.

\section{O método de contornos ativos tradicional}

O método de contornos ativos (MCA) tradicional é baseado em métodos variacionais, cujo objetivo é minimizar uma função representando a energia da curva. Esta curva evolui então, de tal modo que a sua energia diminui a cada nova iteração (Kass et al., 1987; Nixon e Aguado, 2008). O modelo da curva é a parametrização 2D de uma curva geométrica da forma:

$\left\{\begin{array}{l}{[0,1] \rightarrow \mathbb{R}^{2}} \\ s \rightarrow c(s)=[x(s), y(s)]\end{array}\right.$

Este modelo é chamado deformável porque está descrito por uma função energia $E(s)$ que varia em:

$\left\{\begin{array}{l}\mathbb{R}^{2} \stackrel{E(s)}{\longrightarrow} \mathbb{R} \\ c \rightarrow \int_{[0,1]}\left\{\alpha\left|c^{\prime}(s)\right|^{2}+\beta\left|c^{\prime \prime}(s)\right|^{2}+\gamma E_{\text {ext }}[c(s)]\right\} d s\end{array}\right.$

em que os apóstrofos representam a derivação e $E_{\text {ext }}$ é o termo da energia associado com as forças externas (Kass et al., 1987).

A energia é a característica própria do snake (curva) que depende somente de sua forma e da localização de seus pontos. Esta energia é composta geralmente pela energia interna e a energia externa, ou seja, $E=E_{\mathrm{i}}+E_{\mathrm{e}}$ (Bouhours, 2006; Sonka et al., 2007). A energia interna, por sua vez, é geralmente composta também de duas energias $E_{1}$ e $E_{2}$. A primeira $E_{1}=\int \alpha\left|c^{\prime}(s)\right|^{2} d s$ está ligada à elasticidade. Isto significa que esta exprime a faculdade para cada ponto do snake de se afastar de seus vizinhos. A minimização desta energia favorece a busca por pontos, em que o valor de $c^{\prime}(s)$ seja pequeno, isto é, esses pontos tendem a se aproximar e o snake a se concentrar.

A segunda energia $E_{2}=\int \beta\left|c^{\prime \prime}(s)\right|^{2} d s$ é a energia de suavização ou curvatura do snake. Para entender a influência desta componente, pode-se verificar que seu mínimo ocorre quando $c^{\prime \prime}(s)=0$, ou seja, quando $c(s)=k s$ ( $k$ é uma constante), isto é, quando $c$ é uma reta. Assim, favorecer o coeficiente $e_{2}$ durante a fase de minimização, força o snake a diminuir a sua curvatura.

A energia externa permite ao snake uma adaptação sobre o contorno de objetos. É a energia que compensa as outras e evita, assim, que o snake se contraia sobre si mesmo, sem perceber os contornos da imagem. Em geral, a energia externa é calculada utilizando o quadrado do gradiente $\nabla I(x, y)$ da imagem no ponto $(x, y)$ do snake, isto é (Kass et al., 1987):

$$
E_{\text {ext }}(x, y)=-|\nabla[I(x, y)]|^{2}
$$

e também

$$
E_{\text {ext }}(x, y)=-\left|\nabla\left[G_{\sigma} \cdot I(x, y)\right]\right|^{2}
$$

em que $\nabla$ é o operador gradiente e $G_{\sigma}$ é uma gaussiana centrada no ponto $(x, y)$ de variância $\sigma^{2}$. A gaussiana tem como objetivo espalhar a influência da energia ao longo da vizinhança do ponto em que é aplicada. 


\section{Snakes radiais}

Um dos trabalhos mais conhecidos e relevantes sobre snakes radiais ou métodos de contornos ativos radiais (MCAR) é conhecido como Active Rays (Denzler e Niemann, 1999; Denzler et al., 1996). Esta técnica é aplicada em rastreamento de contornos de objetos em tempo real. A idéia é definir um ponto de origem dentro de um contorno e encontrar pontos que o caracterizam, efetuando busca ao longo de raios que divergem a partir de uma origem central $m$. Para isto, as equações de contornos ativos são adaptadas, sendo que o contorno $c(s)$ passa a ser definido por:

$$
\left\{\begin{array}{l}
{[0,1] \rightarrow \mathbb{R}^{2}} \\
s \rightarrow c(s)=c_{m}[\theta(s), r(s)]
\end{array}\right.
$$

em que $c(s)$ é o contorno ativo e $c_{\mathrm{m}}(s)$ é o contorno definido a partir da origem $m$, em coordenadas polares $(\theta, r)$. Neste caso, a energia interna do contorno é calculada pela equação (Denzler et al., 1996):

$$
E_{i}\left[c_{m}(\theta)\right]=\alpha(\theta)\left|\frac{d}{d \theta} r(\theta)\right|^{2}+\beta(\theta)\left|\frac{d^{2}}{d \theta^{2}} r(\theta)\right|^{2}
$$

em que $\alpha(\theta)$ e $\beta(\theta)$ são constantes reais para determinado ângulo $\theta$. O primeiro termo desta equação é a definição da energia de continuidade e o segundo termo, a energia de curvatura para os active rays. Observa-se pela referida equação que estas energias podem ser calculadas ao longo de um feixe. Sendo assim, os cálculos efetuados são unidimensionais.

Outro trabalho relevante é o conhecido como contornos ativos radiais ótimos, que utiliza programação dinâmica na busca da otimização da energia do contorno, e sua principal aplicação é também rastreamento de objetos. A definição da energia total $E$ do contorno é dada por (Chen et al., 2001):

$$
E\left[r_{m}(\theta)\right]=\int_{0}^{2 \pi}\left\{E_{i}\left[r_{m}(\theta)\right]+E_{e}\left[r_{m}(\theta)\right]\right\} d \theta
$$

em que $r_{\mathrm{m}}(\theta)$ é a distância da origem $m$ para o contorno, considerando-se o ângulo $\theta ; E_{\mathrm{i}}$ é a energia interna e $E_{\mathrm{e}}$, a energia externa. A energia externa $E_{\mathrm{e}}$ do contorno ativo é função do gradiente da imagem a ser segmentada, dada por (Chen et al., 2001):

$$
\begin{aligned}
E_{e}\left[r_{m}(\theta)\right]= & \alpha_{e} \cdot g\left[-\left|\frac{d}{d r} \rho_{m}(\theta, r)\right|^{2}\right]= \\
& \alpha_{e} \cdot g\left\{-\left[\rho_{m}(\theta, r+1)-\rho_{m}(\theta, r)\right]^{2}\right\}
\end{aligned}
$$

em que $r$ e $\theta$ são as coordenadas polares de um ponto de controle (o próprio nó) do contorno ativo; $g$ é uma função não linear monotonicamente crescente e $\rho_{m}$ é o feixe (active ray). A energia de continuidade $E_{i}^{c o n t}$ no i-ésimo nó do contorno ativo é calculada pela expressão (Chen et al., 2001):

$$
E_{i}^{\text {cont }}\left[r_{m}(\theta)\right]=\alpha_{i} \cdot\left|r_{m}\left(\theta_{i}\right)-r_{m}\left(\theta_{i-1}\right)\right|^{2}
$$

em que $\alpha_{i}$ é uma constante real.

A energia de curvatura $E_{i}^{\text {curv }}$, por sua vez, é dada pela equação (Chen et al., 2001):

$E_{i}^{\text {curv }}\left[r_{m}(\theta)\right]=$

$\beta_{i} \cdot\left[r_{m}\left(\theta_{i}\right)-r_{m}\left(\theta_{i-1}\right)\right]-\left.\left[r_{m}\left(\theta_{i-1}\right)-r_{m}\left(\theta_{i-2}\right)\right]\right|^{2}$

em que $\beta_{\mathrm{i}}$ é uma constante real.

Outra aplicação em imagens de ultrassom já desenvolvida e que utiliza as técnicas explanadas é a segmentação de artérias, cuja solução é embarcada em DSP (Gemignani et al., 2004, 2007). Uma aplicação encontrada na literatura recente é a segmentação de microcalcificações em mamografias (Arikidis et al., 2008).

Outra aplicação de contorno ativo radial é efetuada em imagens cardíacas de ressonância magnética (MRI), cujo objetivo é a segmentação do ventrículo esquerdo. Além da aplicação em MRI, esse trabalho apresenta uma importante contribuição que consiste na introdução de um campo vetorial gradiente (GVF) no cálculo da energia externa, com bons resultados (Liang et al., 2008).

\section{A transformada Hilbert}

A transformada Hilbert (TH) de uma função real é definida por:

$$
\hat{f}(t)=\frac{1}{\pi} P \int_{-\infty}^{\infty} \frac{f(\tau)}{t-\tau} d \tau
$$

em que $P$ denota o valor principal de Cauchy, já que há uma singularidade na integral para $t=0$. Outra forma de se apresentar a TH é por meio da integral da convolução como (Johansson, 1999; Poularikas, 2000):

$$
\hat{f}(t)=\frac{1}{\pi t} \cdot f(t)
$$

A transformada de Fourier (TF) da função $\frac{1}{\pi t}$ é dada por $\mathfrak{S}\left(\frac{1}{\pi t}\right)=-j \cdot \operatorname{sgn}(\omega)$, em que a função $\operatorname{sgn}(\omega)$ é dada por

$$
\operatorname{sgn}(\omega)=\left\{\begin{array}{l}
+1, \text { se } \omega>0 \\
0, \text { se } \omega=0 \\
-1, \text { se } \omega<0
\end{array}\right.
$$

Dessa forma, com base nestas últimas relações, a TH é normalmente implementada por meio da aplicação da transformada inversa de Fourier (TIF) sobre o resultado da multiplicação de $-j \cdot \operatorname{sgn}(\omega)$ pela TF de $f(t)$, ou seja, $\hat{f}(t)=\mathfrak{I}^{-1}[-j \cdot \operatorname{sgn}(\omega) \cdot \mathfrak{I}(\omega)]$.

Uma característica importante da TH é ser empregada como detector de bordas em sinais, mesmo na presença de ruídos. Assim, esta transformada 
tem sido aplicada especialmente na detecção de alterações bruscas em sinais biomédicos (1D), por exemplo, exames de cardiotocográficos e de ECG (Marques et al., 2008; Madeiro et al., 2008).

\section{Método de contornos ativos radial pSnakes}

O método descrito nesta seção, denominado pSnakes, utiliza as coordenadas polares $(r, \theta)$, em que $r$ é o raio e $\theta$ é o ângulo para a representação dos pixels de uma dada imagem na modalidade ultrassom (Alexandria et al., 2009). A origem dos eixos de referência é localizada no ponto de divergência dos feixes de ultrassom, ou seja, no ponto onde se localiza a sonda ultrassônica.

Seja uma dada imagem digital de entrada $I_{r, \theta}$ em coordenadas polares, representada em forma matricial. Sua dimensão é representada pela quantidade de linhas $R$ e colunas $T$, dados estes que definem a resolução da imagem $R \times$ T. As linhas correspondem aos ângulos dos feixes ultrassônicos e as colunas aos raios, ou seja, distâncias radiais.

\section{Definição do pSnakes}

O MCA pSnakes é um método de contornos ativos radial (MCAR) que pode ser utilizado na segmentação de objetos em imagens digitais, sendo definido por

$$
\left\{\begin{array}{l}
{[0,1] \rightarrow \mathbb{R}^{2}} \\
s \rightarrow c(s)=\left\{\left[r_{1}(s), \theta(s)\right] ;\left[r_{2}(s), \theta(s)\right]\right\}
\end{array}\right.
$$

em que $\left(r_{1}, \theta\right)$ e $\left(r_{2}, \theta\right)$ são as coordenadas polares de pontos de controle (nó) do contorno ativo polar (pSnake). Vale salientar que $r_{1}, r_{2} \subset\left[0, r_{\max }\right] \mathrm{e}$ $\theta(s) \subset\left[\theta_{\min }, \theta_{\max }\right]$, sendo que $r_{\max }$ é o raio de maior alcance dos feixes ultrassônico ou similar; $\theta_{\min }$ e $\theta_{\max }$ são os ângulos limites em que as coordenadas radiais são definidas ou os ângulos limites da deflexão do feixe mencionado. Entretanto, nesta definição são considerados dois pontos $\left(r_{1}, \theta\right)$ e $\left(r_{2}, \theta\right)$. Isso implica que, para cada coordenada angular $\theta$, há dois nós nas posições $r_{1}$ e $r_{2}$ denominados nós gêmeos. Se $\theta_{\text {min }}=0 \mathrm{e}$ $\theta_{\max }=2 \pi$, tem-se o caso especial denominado snakes radial dual. Na Figura 1 são mostrados aspectos geométricos da constituição dos snakes, snakes radiais, pSnakes e snakes radial dual que facilitam a compreensão das principais diferenças entre os métodos mencionados. Observam-se nesta figura o contorno a ser segmentado e a curva (snake) com seus pontos de controle.

O método pSnakes usa um sistema de coordenadas polares definido na Figura 2 e utilizado na localização dos pontos de controle, sendo que $r$ (raio) é a distância de um ponto de controle (nó $P_{1}$ ou $P_{2}$ ) ao referido ponto 0 , enquanto $\theta$ é o ângulo entre o eixo horizontal $x \geq 0$ e o segmento formado pelo nó e o ponto 0 .

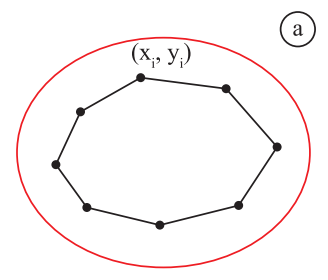

(a)

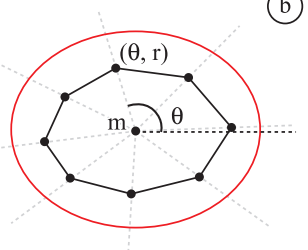

(c)

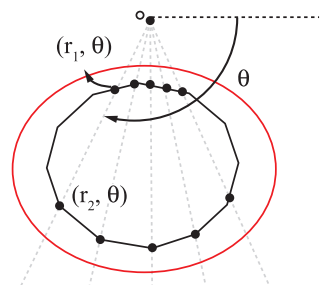

(d)

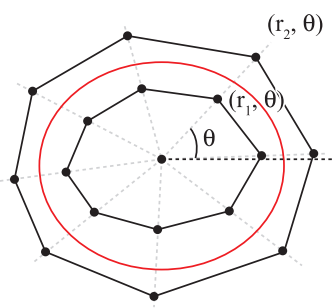

Figura 1. Aspectos geométricos da constituição dos métodos a) snakes, b) snakes radiais, c) pSnakes e d) snakes radial dual.

Figure 1. Geometric aspects about the constitution of the methods a) snakes, b) radial snakes, c) pSnakes and d) dual radial snakes.

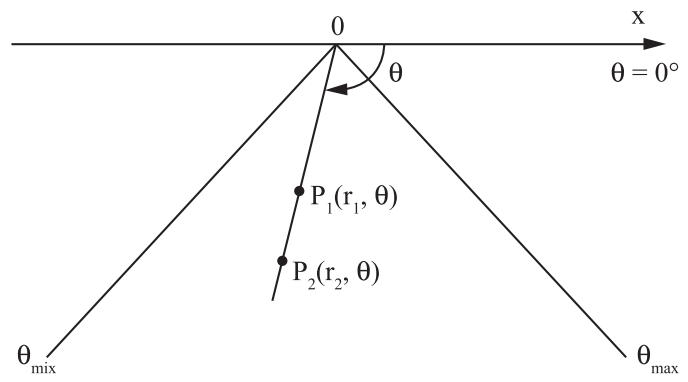

Figura 2. Sistema de coordenadas polares para o pSnakes.

Figure 2. Polar coordinates system for pSnakes.

Para melhor entendimento do funcionamento do pSnakes, um fluxograma do método é apresentado na Figura 3. Inicialmente a imagem a ser segmentada é adquirida. Esta imagem pode ser oriunda de uma conversão de coordenadas cartesianas para polares ou uma imagem sintética ou mesmo uma imagem obtida de um ecocardiógrafo diretamente em coordenadas polares. A partir da imagem de entrada, o contorno inicial é definido manualmente ou por meio de um método automático. Em seguida, novos nós são adicionados ou eliminados de acordo com critérios pré-estabelecidos. A energia total do contorno atual, composta pelas energias interna e externa, é calculada. Após este cálculo, novos posicionamentos para os nós do contorno são buscados de forma a minimizar a energia total do contorno. Para essa finalidade, um algoritmo de otimização específico é utilizado. Se um critério de parada é atingido, o método pSnakes chega ao fim de sua aplicação, resultando na imagem segmentada, constituída pelos últimos cálculos dos nós. 


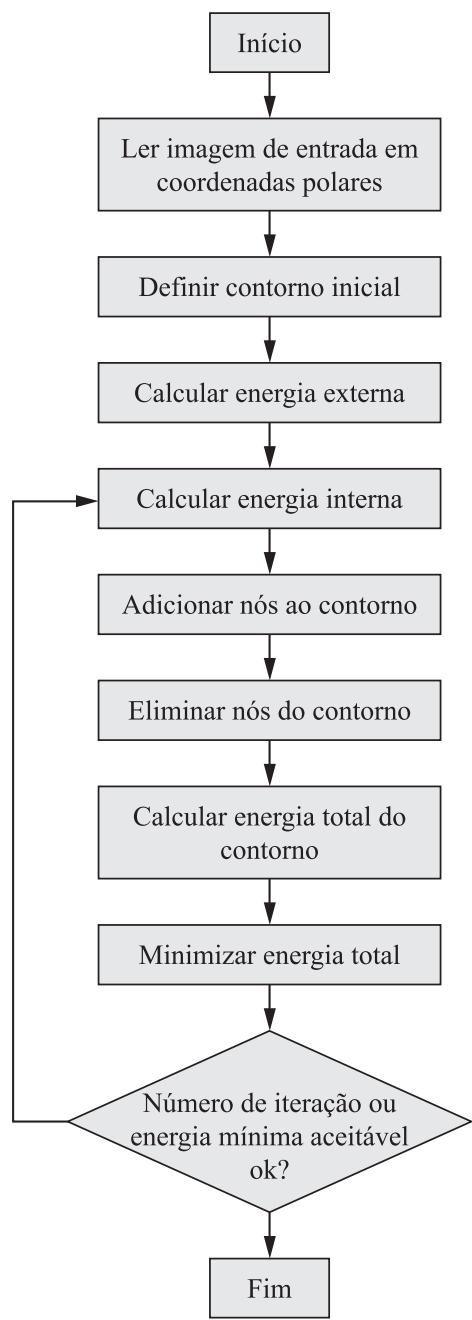

Figura 3. Fluxograma explicativo do método pSnakes. Figure 3. Functional flowchart for pSnakes method.

Em caso contrário, nova iteração é realizada. Os critérios de parada normalmente utilizados são número de iterações ou energia total mínima aceitável.

Os métodos de contornos ativos, em geral, inclusive os radiais, como o pSnakes possuem sua dinâmica baseada na minimização da energia total que é constituída das energias interna e externa.

\section{Energias internas}

A energia é a principal característica descritiva do snake e a energia interna depende somente da forma e localização dos pontos do contorno ativo e, em geral, está relacionada com a curvatura $E_{\text {curv }_{v e} \text { e a }}$ continuidade $E_{\text {cont } t: \theta^{-\theta}}$ do contorno, sendo dada pela soma destas, ou seja, $E_{i}=E_{\operatorname{curv}_{r} \theta}+E_{\text {cont }_{t} \text {. }}$.

A energia de curvatura polar é calculada por (Chen et al., 2001):

$$
E_{\text {curv } r, \theta}=\left[\left(r_{\theta}-r_{\theta+1}\right)-\left(r_{\theta+1}-r_{\theta+2}\right)\right]^{2}
$$

em que $r_{\theta}$ é a posição radial de um dado nó ao longo do feixe de ângulo $\theta, r_{\theta+1}$ é a posição radial de um outro nó adjacente de coordenada $\theta+1$ e $r_{\theta+2}$ é a posição radial do nó subsequente ao de coordenada $\theta+1$. Assim, quando o contorno tende a ser uma linha reta, essa energia tende a zero. Por outro lado, quando se tem um nó com distância radial maior do que a distância dos vizinhos da esquerda ou direita, o valor da energia de curvatura tende a aumentar nessa vizinhança.

A energia de continuidade polar $E_{c o n t t_{t},}$, por sua vez, é dada por (Chen et al., 2001):

$E_{\text {cont } t_{, \theta}}=\left(r_{\theta}-r_{\theta+1}\right)^{2}$

As equações 15 e 16 são semelhantes às equações 10 e 9 , respectivamente, definidas por Chen et al. (2001). A diferença está no significado das coordenadas polares, já que para os snakes radiais o ponto de origem dos eixos polares está dentro da região a ser segmentada, enquanto no método $\mathrm{pSnakes}$ o referido ponto está geralmente fora desta região.

A equação 15 é baseada na segunda derivada do raio em relação ao ângulo $\theta$. Na equação 16 , a energia de continuidade é calculada tomando por base a diferença da coordenada radial $r$ do nó e a do seu vizinho. Essas equações são escolhidas na definição da energia interna por já serem utilizadas na literatura.

\section{Transformada de Hilbert como energia externa em snakes radiais}

A transformada de Hilbert (TH) possui características que a tornam bastante eficiente quando usadas como energia externa em contornos ativos radiais. Para que isso seja possível, deve-se aplicar a TH 1D ao longo dos feixes radiais da imagem representada em coordenadas polares. Para ser utilizada como energia externa, sugere-se que a TH seja normalizada e tomado seu valor absoluto. Assim, propõe-se que a energia externa em contornos ativos radiais seja dada por (Alexandria et al., 2009, 2010):

$$
E_{\text {ext }}(r, \theta)=|\hat{f}(r)|
$$

que pode ser normalizada pela expressão:

$$
E_{\text {ext }}^{n}(r, \theta)=1-\frac{|\hat{f}(r)|}{\max \left(\left|\hat{f}\left(r_{i}\right)\right|\right)}
$$

para $r_{i} \in\left[0, r_{\text {max }}\right]$.

Caso se considere que o ponto de divergência dos feixes em snakes radiais esteja no interior do objeto a ser segmentado, a TH tende a atingir valores 
negativos próximos à borda de subida e valores positivos após a borda de descida. Também se pode empregar a expressão para o cálculo da energia externa, desprezando os valores na região que está fora do contorno do objeto, ou seja:

$$
E_{\text {ext }}^{n}(r, \theta)=\left\{\begin{array}{l}
1-\frac{|\hat{f}(r)|}{\max \left(\left|\hat{f}\left(r_{i}\right)\right|\right)}, \text { se } \hat{f}(r)<0 \\
0, \text { se } \hat{f}(r) \geq 0
\end{array}\right.
$$

O módulo (ou valor absoluto) da TH normalizada da intensidade dos pixels ao longo de um feixe é chamado de campo hilbertiano ou energia hilbertiana e é utilizado como energia externa no pSnakes. Dessa forma, a energia externa hilbertiana é simplesmente definida como o módulo (ou valor absoluto) da TH normalizada, aplicada ao longo do sinal de um feixe, ou seja, ao longo do raio.

Um caso típico do comportamento da energia hilbertiana é ilustrado na Figura 4. O vale mostrado na Figura $4 \mathrm{~b}$ corresponde à borda do objeto mostrado na Figura 4a. Conforme mostrado nesta figura, a intensidade do campo aumenta à esquerda do vale mencionado, servindo de guia na busca da energia mínima do snake, correspondendo à segmentação desejada. Assim, utilizando-se as equações 18 ou 19 para calcular a energia externa, um nó (ponto de controle) do snake radial, sob ação dessa energia, tende a se aproximar das bordas do objeto.

A energia externa hilbertiana para ser utilizada no pSnakes é definida conforme a equação 18 . Com base nas definições anteriores de energia hilbertiana, a energia total do pSnakes é dada por:

$$
p E_{\text {total }_{r, \theta}}=\alpha \cdot E_{\text {curv }_{r, \theta}}+\beta \cdot E_{\text {cont }_{r, \theta}}+\gamma \cdot E_{\text {ext }_{r, \theta}}
$$

em que $\alpha, \beta$ e $\gamma$ são constantes reais $E_{\text {ext }}$ é a energia externa hilbertiana. Um exemplo da energia hilbertiana ao longo de um feixe passando por uma cavidade é apresentado na Figura 5.

\section{Dinâmica do pSnakes}

A dinâmica do pSnakes é realizada por meio de sua expansão ou retração e inclusão ou eliminação de nós. A expansão ou encolhimento ocorre principalmente pela movimentação dos nós no sentido radial, visando à minimização da energia total $p E_{\text {total }}$ do contorno. Para isto, pode-se utilizar o algoritmo greedy (Demaine e Leiserson, 2001; Bednorz, 2008) ou programação dinâmica (Chen et al., 2001), entre outros algoritmos de otimização. O método de otimização adotado neste trabalho é o greedy.

A inclusão e exclusão de nós também são elementos importantes na dinâmica do pSnakes. A inclusão permite um maior refinamento do contorno e, portanto,
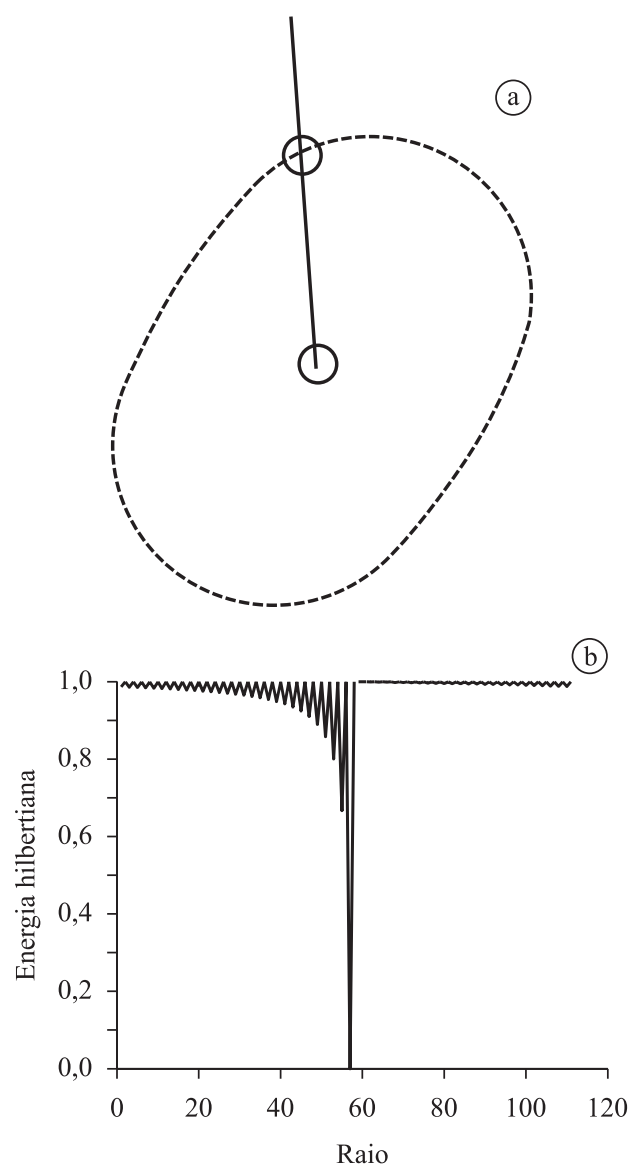

Figura 4. Energia hilbertiana ao longo de um feixe passando por uma cavidade - situação típica, a) feixe atravessando o contorno do objeto e b) energia hilbertiana ao longo do feixe.

Figure 4. Hilbertian energy along a beam passing through a cavity - typical situation, a) beam passing through the object's edge and b) Hilbertian energy along the beam.

maior acurácia na segmentação dos contornos de objetos. Com comportamento contrário à inclusão, a exclusão elimina os nós que estão em discrepância com o restante do contorno, baseada em critérios da intensidade de energia no nó. Isto ocorre principalmente na presença de ruído em imagens a serem segmentadas. Para isso, algumas constantes configuráveis são consideradas no processo de adição e deleção de nós: $n$ (número de vizinhos do nó investigado); $L_{\text {inc }}$ (limiar de energia para inclusão de nó); $L_{\text {lat }}$ (limiar de energia para inclusão de nó no limite lateral do contorno); $\theta_{\text {lat }}$ (ângulo lateral de inserção) e $L_{\text {exc }}$ (limiar de energia para exclusão de nó).

Um nó gêmeo deve ser inserido entre dois nós gêmeos já existentes se a média da energia de curvatura de $n$ vizinhos próximos, calculada para o nó gêmeo 1 ou 2, é maior do que um dado limiar $L_{\text {inc }}$ (limiar de inclusão). O fator $n$ é calculado 

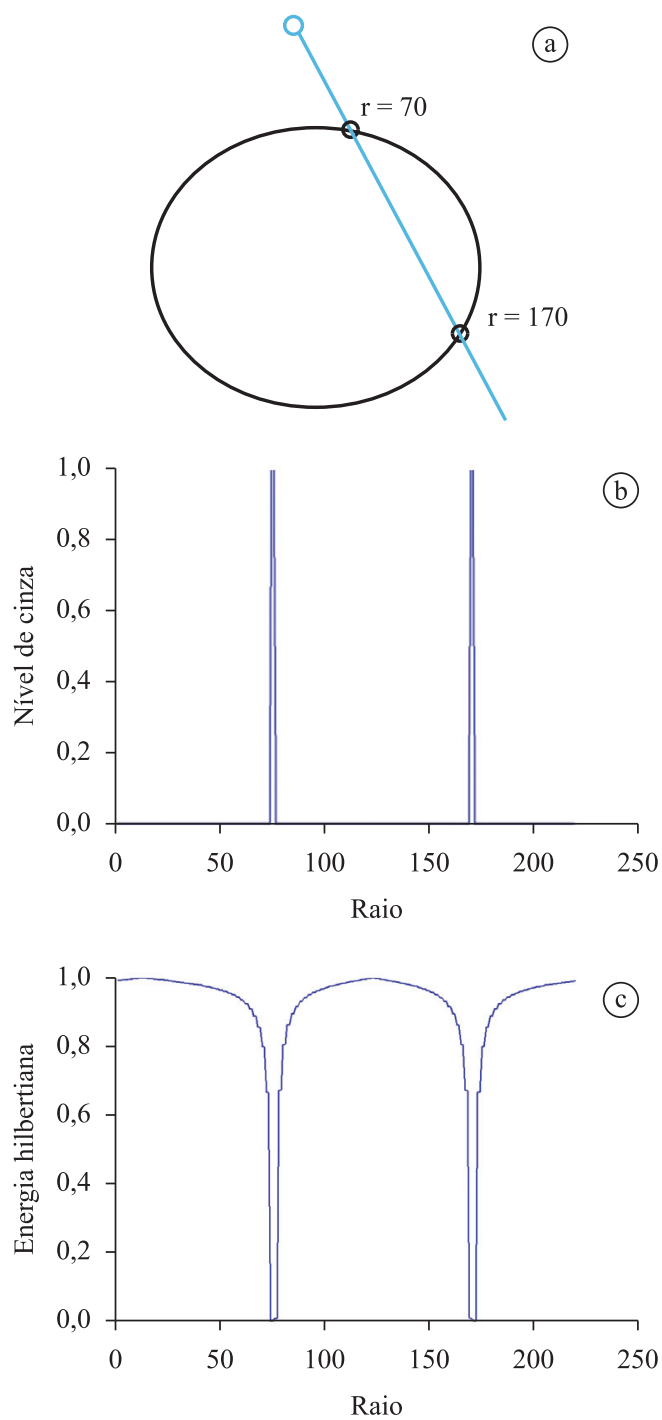

Figura 5. Exemplo da energia hilbertiana ao longo de um feixe; a) feixe passando por uma cavidade, b) intensidade dos pixels ao longo do feixe e c) energia hilbertiana.

Figure 5. Example of Hilbertian energy along a beam; a) active ray passing through a cavity, b) pixel intensity along the beam and c) Hilbertian energy.

automaticamente tomando-se um percentual da quantidade de nós (por exemplo, 10\%) no pSnake completo. Cada nó gêmeo incluído, cujas coordenadas são definidas como o raio e ângulos médios de seus vizinhos.

Nota-se que há uma tendência do $p$ Snake em não crescer para as laterais, caso se adicione somente nós entre os nós já existentes. Assim, torna-se necessária a inclusão de regras especiais para os limites laterais do contorno. Isto é realizado por meio da verificação dos nós gêmeos mais próximos do limite angular esquerdo e direito. Se a distância entre estes é maior do que um dado limiar lateral $L_{l a t}$, inclui-se um nó gêmeo a mais no extremo lateral (esquerdo ou direito). Neste caso a distância a ser inserida é de um ângulo $\theta_{\text {lat }}$ (ângulo lateral de inserção). Um nó gêmeo deve ser excluído do pSnake, se a média da energia de continuidade é menor que um dado limiar $L_{e x c}$ (limiar de exclusão), em ambos os nós gêmeos, para $n$ vizinhos próximos.

Essas regras visam um balanceamento entre a representação de detalhes no contorno a ser segmentado da imagem e a diminuição de erros provocados por ruído ou por falhas no contorno. Uma forma alternativa utilizada, como parâmetro de decisão, para inclusão e exclusão de nós é o desvio padrão da energia de curvatura e de continuidade. Esta forma alternativa mencionada não é utilizada neste trabalho.

\section{Fração de ejeção do ventrículo esquerdo}

Vários parâmetros para avaliação da função cardíaca, em especial medidas relativas ao ventrículo esquerdo são usadas em medicina. Dentre estas, destaca-se a fração de ejeção (FEJ) que consiste na comparação entre os volumes finais da diástole (VFD) e da sístole (VFS) e é dada por Otto (2004):

$$
\mathrm{FEJ}=\frac{(V F D-V F S)}{\mathrm{VFD}} \cdot 100 \%
$$

\section{Materiais}

O método pSnakes foi implementado e as simulações foram realizadas utilizando-se o ambiente de desenvolvimento e simulação MATLAB ${ }^{\circledR}$, versão 7.6 r2008a, rodando em um notebook com processador Intel ${ }^{\circledR}$ Core $^{\mathrm{TM}} \mathrm{i} 5$, memória $3 \mathrm{~GB}$, com sistema Windows ${ }^{\circledR} 7$ Home Premium instalado. Na primeira etapa, os testes são realizados visando uma verificação preliminar da eficácia do pSnakes na segmentação de imagens sintéticas e reais. As imagens sintéticas utilizadas possuem objetos no formato de triângulo, retângulo e círculo. A segunda etapa de testes foi feita com imagens reais de ecocardiograma. Foram usados dezessete exames de ecocardiograma, totalizando 34 imagens, entre imagens de fim de diástole e final de sístole. Estas imagens foram obtidas de um ecocardiógrafo (GE, modelo Vivid 7 PRO) da Clínica Prontocárdio em Fortaleza, CE. Um ecocardiografista (ECO1) registra dezessete exames sincronizados pelo eletrocardiograma (ECG), na posição de eixo curto do ventrículo esquerdo (VE). Por meio do trackball efetua-se manualmente os contornos das bordas internas no final da diástole $\mathrm{e}$ sístole do VE, sincronizados com o início da onda Q e pico da onda T do ECG, respectivamente, com obtenção imediata de volume diastólico, sistólico e fração de ejeção (FEJ). Outro ecocardiografista 
(ECO2) executa, de forma independente, as medidas das mesmas 34 imagens em computador portátil para análise interobservador. O cálculo dos volumes é obtido empregando-se a regra de Simpson (Otto, 2004). O método de segmentação automática pSnakes é aplicado nas medidas dos grupos ECO1 e ECO2 para comparação posterior. A fração de ejeção é calculada para cada exame e para cada um dos três métodos utilizados: ECO1, ECO2 e pSnakes. A inicialização do pSnakes é realizada manualmente próxima das bordas e constituída de três nós gêmeos, através de dispositivo apontador.

Em seguida a fração de ejeção é novamente calculada utilizando-se os métodos de contornos ativos tradicional (ST) (Cohen, 1991; Dagher e Tom, 2008), snakes radial com derivada (SRD), snakes radial hilbertiano (SRH) e pSnakes, para inicializações diferentes: na borda e 30\%, 50\%,70\% mais distante da borda. O SRH consiste no snakes radial empregando-se a energia hilbertiana como energia externa. A média e desvio padrão da fração de ejeção são obtidos, assim como a correlação em relação a ECO1, por ser o mais experiente dos dois ecocardiografistas.

\section{Resultados}

Os resultados neste trabalho estão dispostos em duas etapas. A primeira etapa corresponde a aplicação do pSnakes em imagens sintéticas e na segunda etapa é realizada a segmentação em imagens de ecocardiograma no cálculo de fração de ejeção.

\section{Resultados para imagens sintéticas}

$\mathrm{Na}$ primeira etapa, as imagens sintéticas utilizadas para os testes mencionados são apresentadas nas Figuras 6a-c, em que se pode observar o ponto de origem dos feixes (active rays), localizado na posição $(x=170, y=1)$, nas imagens de teste (em vermelho).

Nestes testes, as equações 16 e 15 são utilizadas como expressões para o cálculo das energias internas. Para cálculo da energia total do contorno ativo, emprega-se a equação 20 , com valores para as constantes de $\alpha=\beta=\gamma=1$. A energia externa
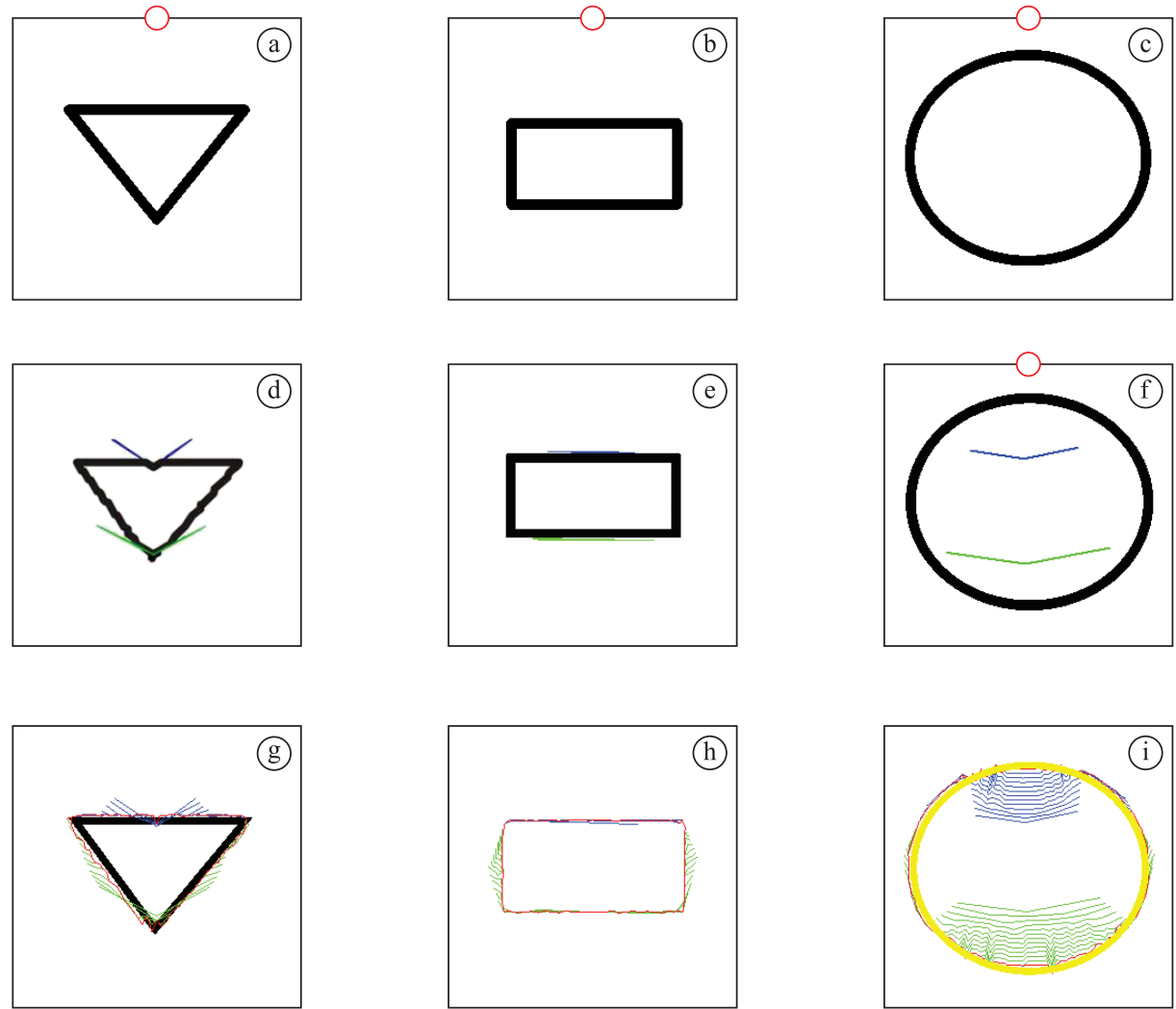

Figura 6. Imagens de teste para avaliação do pSnakes: a) triângulo, b) retângulo e c) círculo. Contorno inicial para cada imagem de teste em d), e) e f). Dinâmica de convergência do pSnakes para as imagens de teste em g), h) e i).

Figure 6. pSnakes test images: a) triangle, b) rectangle and c) circle. Initial contour for each test image in d), e) and f). Initial contour for each test image in $g$ ), $h$ ) and $i$ ). 
utilizada é a energia hilbertiana normalizada, conforme equação 19. Para a minimização da energia total do snake radial, usa-se o algoritmo greedy (Bednorz, 2008), com espaço de busca ao longo do raio composto pela vizinhança 7 , centrado no ponto atual.

Para aplicação do método pSnakes são utilizados 120 feixes de 220 pixels de raio. O snake inicial é obtido por meio de seleção manual de 3 nós gêmeos, utilizando um dispositivo apontador mouse sobre a própria imagem de entrada. Os pontos que definem os contornos iniciais usados para cada imagem de teste são mostrados nas Figuras 6d-f. Em azul observam-se os nós $r_{1}(s)$ e em verde, os nós $r_{2}(s)$, conforme definição do pSnakes. São realizadas trinta (30) iterações para convergência do algoritmo e o resultado final de segmentação para cada imagem de teste é apresentado na Figuras 6g-i. Observa-se que o contorno ativo acompanha a borda do objeto a ser segmentado, apesar de apresentar alguma falta de acurácia na segmentação.

A energia hilbertiana para cada exemplo é mostrada na Figura 7. Considerando que quanto mais claros os tons das cores (em especial o azul) na referida figura, maior é a intensidade da energia hilbertiana, observa-se que, à medida que se afasta das bordas do objeto a ser segmentado, a energia cai suavemente e sua influência ainda se mantém, mesmo longe da borda do objeto.

Os resultados obtidos das segmentações com o pSnakes para as imagens de teste são apresentados, em coordenadas polares, na Figura 8. Os círculos "O" (amarelo) e os "X" (branco) na figura correspondem aos nós do pSnake, $r_{1}(s)$ e $r_{2}(s)$, respectivamente.

\section{Resultados para imagens de ecocardiograma no cálculo de fração de ejeção}

Os resultados obtidos para a fração de ejeção para as imagens de ecocardiograma de eixo curto são mostrados na Tabela 1 . A configuração dos parâmetros do pSnakes utilizada é de: $\alpha=0,1, \beta=0,2$ e $\gamma=1$. Na média dos dezessete exames obteve-se um desvio da medida da fração de ejeção do ventrículo esquerdo de $10 \pm 7 \%$, utilizando-se o método pSnakes em relação à medição manual do ecocardiografista $\mathrm{ECO} 1$, e $10 \pm 9 \%$, em relação a ECO2. O erro interobservador entre as medidas dos ECO1 e ECO2 é de $9 \pm 6 \%$.

Para a análise estatística é aplicado teste- $t$ com nível de significância $p<0,05$. Sendo assim, a FEJ não é estatisticamente diferente na comparação entre os grupos ECO1 versus ECO2 (68 $\pm 8 \%$ versus $70 \pm 11 \% ; \mathrm{p}=0,248)$, ECO1 versus $\mathrm{pSnakes}$ ( $68 \pm 8 \%$ versus $71 \pm 12 \% ; \mathrm{p}=0,136)$ e $\mathrm{ECO} 2$ versus pSnakes $(70 \pm 11 \%$ versus $71 \pm 12 \% ; \mathrm{p}=0,355)$.
Um exemplo de segmentação de uma imagem de ecocardiograma em eixo curto é apresentado na Figura 9. Observa-se na Figura 9a a imagem original obtida ao final da sístole. A segmentação manual de ECO1 por um especialista médico é mostrada na Figura 9b. A segmentação obtida pelo método pSnakes é apresentada na Figura 9c. Nesta última figura pode-se observar, em azul, a presença dos nós $r_{1}(s) \mathrm{e}$, em verde, os nós $r_{2}(s)$, de acordo com a definição de pSnakes.

Os resultados obtidos comparando-se o cálculo de fração de ejeção obtidos pelo pSnakes, pelos resultados de ST, SRD e SRH são expostos na Tabela 2.

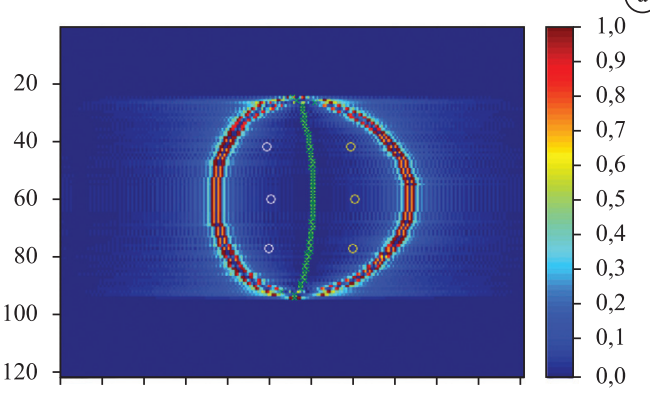

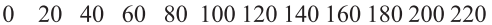
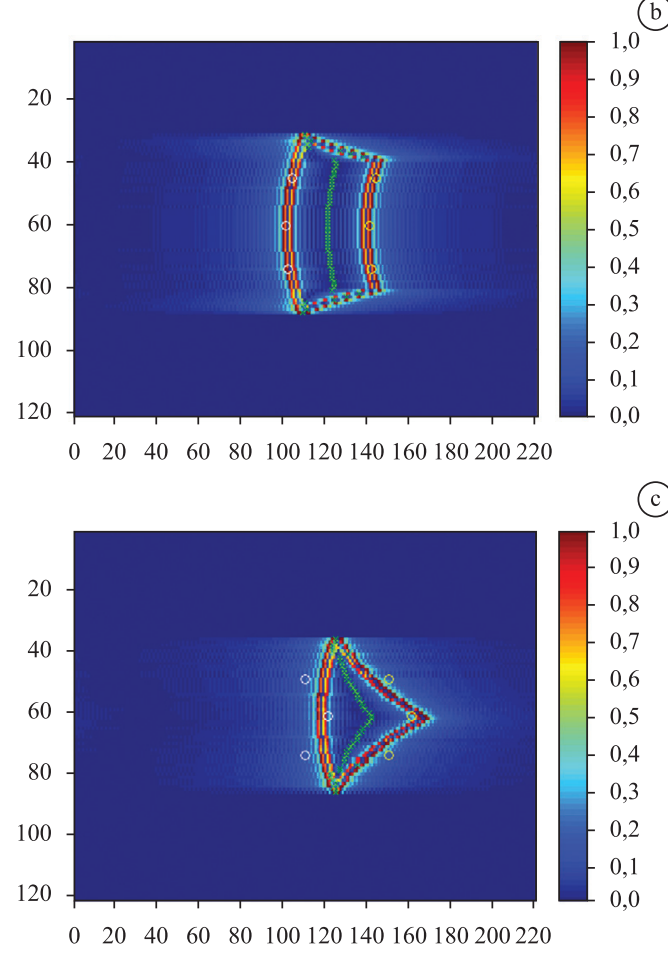

Figura 7. Energia hilbertiana para cada imagem de teste para as imagens de teste: a) triângulo, b) retângulo e c) círculo.

Figure 7. Hilbertian energy for each test image, for the test images: a) triangle, b) rectangle and c) circle. 


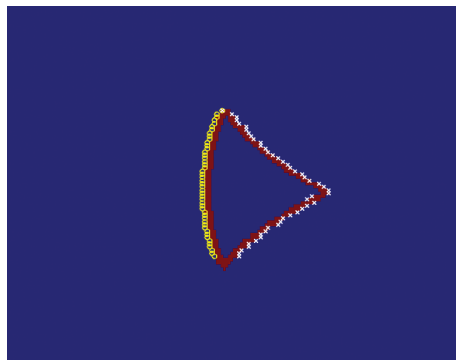

(a)

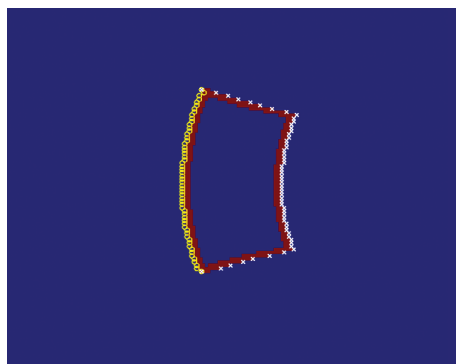

(b)

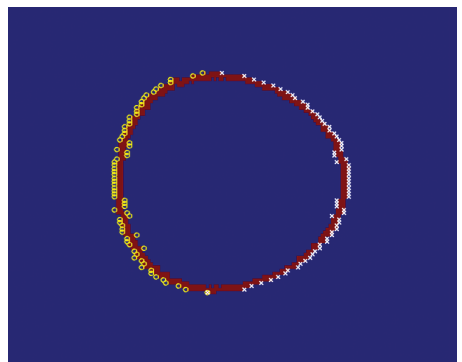

(c)

Figura 8. Resultados obtidos apresentados em coordenadas polares, para as imagens de teste: a) triângulo, b) retângulo e c) círculo. Figure 8. Obtained results presented in polar coordinates, for the test images: a) triangle, b) rectangle and c) circle.

Tabela 1. Fração de ejeção obtida por ECO1, ECO2 e pSnakes.

Table 1. Ejection fraction obtained by ECO1, ECO2 and pSnakes.

\begin{tabular}{|c|c|c|c|c|c|c|}
\hline \multirow[b]{2}{*}{ Caso } & \multicolumn{3}{|c|}{ Fração de ejeção } & \multicolumn{3}{|c|}{ Desvio } \\
\hline & ECO1 (\%) & ECO2 (\%) & pSnakes (\%) & $\begin{array}{c}\mathrm{ECO} 1 \times \mathrm{ECO} 2 \\
(\%)\end{array}$ & $\begin{array}{c}\text { ECO1 } \times \\
\text { pSnakes }(\%)\end{array}$ & $\begin{array}{c}\text { ECO2 } \times \\
\text { pSnakes }(\%)\end{array}$ \\
\hline A & 73 & 75 & 77 & 3 & 5 & 2 \\
\hline $\mathrm{B}$ & 70 & 79 & 67 & 13 & 4 & 15 \\
\hline $\mathrm{C}$ & 53 & 64 & 60 & 20 & 13 & 6 \\
\hline $\mathrm{D}$ & 73 & 79 & 74 & 8 & 1 & 6 \\
\hline E & 87 & 93 & 89 & 7 & 3 & 4 \\
\hline $\mathrm{F}$ & 70 & 78 & 69 & 11 & 1 & 11 \\
\hline G & 62 & 49 & 69 & 21 & 11 & 40 \\
\hline $\mathrm{H}$ & 59 & 64 & 66 & 9 & 12 & 3 \\
\hline I & 63 & 62 & 72 & 2 & 14 & 17 \\
\hline $\mathrm{J}$ & 75 & 70 & 80 & 7 & 7 & 15 \\
\hline $\mathrm{K}$ & 56 & 47 & 44 & 15 & 22 & 8 \\
\hline $\mathrm{L}$ & 68 & 66 & 72 & 3 & 6 & 9 \\
\hline M & 61 & 61 & 54 & 1 & 12 & 12 \\
\hline $\mathrm{N}$ & 72 & 79 & 89 & 10 & 23 & 12 \\
\hline $\mathrm{O}$ & 71 & 75 & 81 & 6 & 15 & 8 \\
\hline $\mathrm{P}$ & 67 & 75 & 74 & 12 & 10 & 2 \\
\hline \multirow[t]{2}{*}{ Q } & 70 & 74 & 79 & 5 & 12 & 7 \\
\hline & & & & $\mathrm{p}=0,248$ & $\mathrm{p}=0,136$ & $\mathrm{p}=0,355$ \\
\hline Média & 68 & 70 & 71 & 9 & 10 & 10 \\
\hline Desvio padrão & 8 & 11 & 12 & 6 & 7 & 9 \\
\hline
\end{tabular}
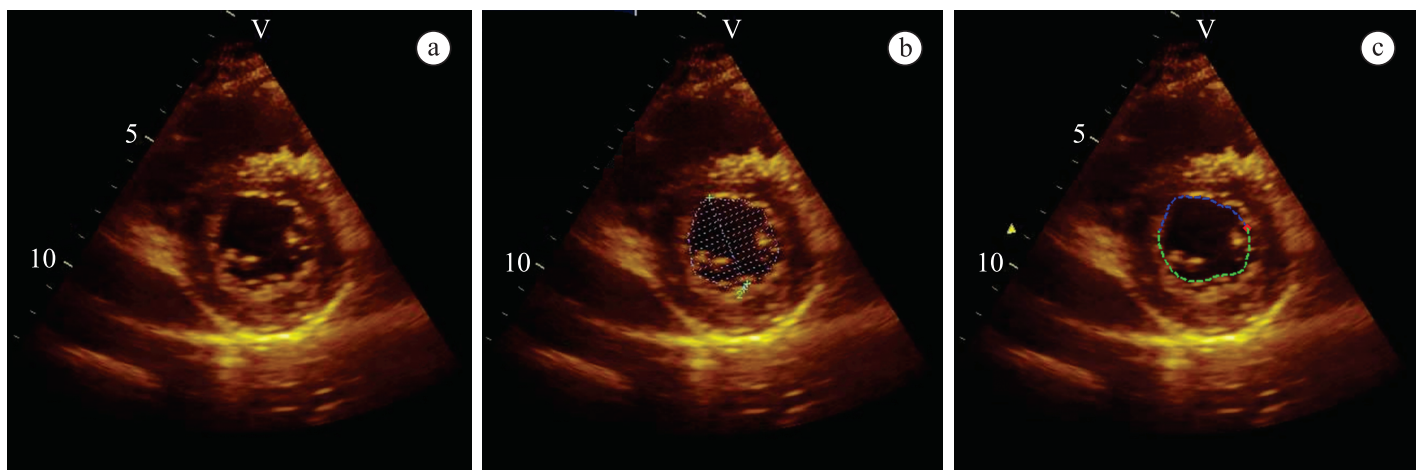

Figura 9. Resultados obtidos para imagem de ecocardiograma em eixo curto: a) imagem original - final de sístole, b) segmentação manual realizada pelo médico ECO1 e c) segmentação obtida pelo pSnakes.

Figure 9. Obtained results for short axis echocardiogram image: a) original image - end of systole, b) physician ECO1 manual segmentation and c) pSnakes segmentation. 
Tabela 2. Fração de ejeção, média e desvio padrão, obtidos por ST, SRD, SRH, pSnakes e ECO1, sobre a borda do VE e 30\%, 50\% e 70\% mais distante da borda.

Table 2. Ejection fraction, average and standard deviation obtained by ST, SRD, SRH, pSnakes e ECO1, on LV border and 30\%, 50\% 70\% more distant from the border.

\begin{tabular}{|c|c|c|c|c|c|c|c|c|c|}
\hline \multirow{2}{*}{ Inicialização } & \multicolumn{2}{|c|}{ ST } & \multicolumn{2}{|c|}{ SRD } & \multicolumn{2}{|c|}{ SRH } & \multicolumn{2}{|c|}{ pSnakes } & \multirow{2}{*}{$\begin{array}{l}\text { ECO1 } \\
\text { Média }\end{array}$} \\
\hline & Média & DP & Média & DP & Média & DP & Média & DP & \\
\hline Próxima à borda & 71,9 & 15,3 & 79,5 & 8,1 & 72,3 & 13,2 & 71,5 & 11,5 & 67,6 \\
\hline $30 \%$ & 68,5 & 9,3 & 79,2 & 9,2 & 70,9 & 15,0 & 72,6 & 9,4 & 67,6 \\
\hline $50 \%$ & 70,7 & 11,9 & 78,9 & 9,2 & 68,6 & 16,2 & 72,1 & 10,8 & 67,6 \\
\hline $70 \%$ & 76,0 & 13,8 & 78,4 & 9,0 & 70,4 & 14,6 & 71,0 & 11,7 & 67,6 \\
\hline Total & 71,8 & 12,6 & 79,0 & 8,9 & 70,6 & 14,7 & 71,8 & 10,9 & 67,6 \\
\hline
\end{tabular}

Todas as comparações são em relação a ECO1. A configuração dos parâmetros utilizada nestes testes para pSnakes é $\alpha=0,1, \beta=0,2$ e $\gamma=1$; para ST é de $\alpha=0,2, \beta=0,2$ e $\gamma=1$; para SRD tem-se $\alpha_{i}=0,1$, $\beta_{i}=0,2$ e $\alpha_{e}=0,5$ e para SHR, $\alpha_{i}=0,2, \beta_{i}=0,2$ e $\gamma=0,5$.

Observa-se que o pSnakes obtém a segunda melhor média, ou seja, segunda média mais próxima de ECO1, com menor desvio padrão $(71,8 \pm 10,9)$. SRH obtém a média mais próxima de ECO1, porém o desvio padrão obtido é o maior $(70,8 \pm 14,7)$. ST com 71,8 $\pm 12,6$ também está próximo de SRH. Por outro lado, o SRD apresenta a média mais afastada de ECO1, porém com o menor desvio padrão $(79,0 \pm 8,9)$. Quanto à correlação $(\rho)$ entre ECO1 e os MCA avaliados, o pSnakes obtém o maior fator em todas as situações, em torno de $\rho=0,80(0,81,0,79,0,83$ e 0,83 , para distância sobre a borda, $30 \%, 50 \%$ e $70 \%$ distante da borda, respectivamente) seguido de longe por SRD e ST, com valores de $\rho$ entre 0,30 e 0,50, conforme é mostrado na Tabela 3. Ainda com base nesta tabela, SRH possui os resultados de menor correlação (abaixo de 0,20 ).

É importante ressaltar que a média e o desvio padrão isoladamente não trazem informações completas sobre os desvios obtidos para cada método avaliado. Porém em conjunto, as duas estatísticas servem como base para a avaliação. Por isso, no conjunto das duas medidas, o pSnakes consegue o melhor resultado. Além disso, a correlação entre pSnakes e ECO1 confirma esta análise já que pSnakes obtém a maior fator de correlação diante dos outros MCA. Pode-se observar ainda que à medida que a inicialização dos MCA se distancia da borda do VE, os resultados de pSnakes e SRH, baseados na transformada de Hilbert, melhoram, enquanto os resultados de ST, MCA baseado no gradiente, pioram. Quanto à questão do ruído, neste trabalho não se efetua diretamente uma análise do desempenho do pSnakes na presença de ruído, mas, indiretamente, ao se inicializar o pSnake e os outros MCA longe da borda ( $70 \%$ mais distante,
Tabela 3. Correlação em relação a ECO1 versus ST, SRD, SRH, pSnakes e ECO1, sobre a borda do VE e 30\%, 50\% e 70\% mais distante da borda.

Table 3. Correlation related to ECO1 versus ST, SRD, SRH, pSnakes e ECO1, on LV border and 30\%, 50\% 70\% more distant from the border.

\begin{tabular}{lcccc}
\hline \multirow{2}{*}{ Inicialização } & \multicolumn{4}{c}{ Correlação - ECO1 versus } \\
\cline { 2 - 5 } & ST & SRD & SRH & pSnakes \\
\hline Próxima à & 0,53 & 0,50 & 0,16 & 0,81 \\
borda & 0,51 & 0,48 & 0,19 & 0,79 \\
$30 \%$ & 0,38 & 0,50 & 0,19 & 0,83 \\
$50 \%$ & 0,48 & 0,40 & $-0,05$ & 0,83 \\
$70 \%$ & & & \\
\hline
\end{tabular}

Tabela 4. Tempo médio de processamento ST, SRD, SRH, pSnakes, 140 iterações.

Table 4. Time processing time for ST, SRD, SRH, pSnakes, 140 iterations.

\begin{tabular}{lc}
\hline & Tempo médio de processamento (s) \\
\hline ST & 1,79 \\
SRD & 0,56 \\
SRH & 0,56 \\
pSnakes & 0,69 \\
\hline
\end{tabular}

por exemplo), tem-se uma incidência de ruído speckle intensa que proporciona um bom teste de robustez a ruído para os MCA. Desta forma, os resultados alcançados sugerem boa robustez a ruído para o pSnakes, em especial em relação ao ruído speckle muito presente em imagens de ecocardiograma, já que se manteve estável em todas as situações testadas.

Quanto à média de tempo de processamento de cada imagem, o ST apresentou pior resultado, enquanto que os métodos radiais se apresentaram melhor, conforme Tabela 4. SRD e SRH demoram em média $0,56 \mathrm{~s}$ em cada imagem de ecocardiograma, enquanto que o pSnakes demorou 0,69 s. ST delongou 1,79 s. Foram consideradas 140 iterações para cada método. Estes resultados já eram esperados, visto que em ST o espaço de busca do valor mínimo da energia total 
do contorno ativo é um espaço $x y$ (2D), espaço este maior que o espaço de busca dos MCAR (1D). Vale salientar que estes tempos de processamento podem ser melhorados ao se otimizar os códigos implementados e utilizar-se ferramentas computacionais mais rápidas como linguagem $\mathrm{C} / \mathrm{C}++$ embarcada em processadores dedicados.

\section{Conclusões}

O MCAR pSnakes é apresentado neste trabalho e avaliado no cálculo de fração de ejeção do ventrículo esquerdo (VE) em imagens de ecocardiograma em eixo curto. As principais contribuições deste trabalho são o uso do MCAR pSnakes no cálculo de FEJ; o emprego da energia hilbertiana, calculada através da TH, como energia externa no MCAR proposto e a avaliação da aplicação do pSnakes e outros MCA no cálculo da fração de ejeção do VE.

A utilização da TH como energia externa em contornos ativos pSnakes, assim como no snakes radial, pelos dados de correlação, média e desvio padrão, mostra-se bastante adequada para a aplicação (segmentação do VE, visando ao cálculo de FEJ). Isto se deve principalmente às características da energia hilbertiana de alcançar um mínimo de energia nas bordas de objetos e sofrer um acréscimo suave em seu valor, perdurando sua influência mesmo longe das bordas.

Uma das contribuições principais desta nova abordagem proposta é esta poder substituir a derivada como energia externa nos MCAR. A derivada, utilizada nos trabalhos de Chen et al. (2001) e Denzler e Niemann (1999) é definida somente muito próxima das bordas, tornando-se difícil o seu uso quando o snake inicial está distante da borda. O emprego da TH também se torna vantajoso em relação à proposta apresentada por Hunter et al. (1995), já que dispensa o uso de máscara gaussiana (filtro) para espalhar a influência da derivada ao longo dos feixes. Além disso, a influência da energia hilbertiana se faz presente a uma distância maior em relação ao método proposto na referência mencionada, permitindo a inicialização do snake radial distante da borda. Outra vantagem da energia hilbertiana é que esta pode ser aplicada em tempo real, já que a transformada de Hilbert 1D possui baixo tempo de processamento. Vale ressaltar que o tempo de processamento obtido pelos métodos radiais e polar neste trabalho foram cerca de três vezes mais rápidos que o método cartesiano ST. Outrossim, o espaço de busca maior ou igual a cinco se mostra capaz de fazer a dinâmica do contorno ativo imune às variações na intensidade da $\mathrm{TH}$, conforme é ilustrado na Figura 5
Sistemas baseados no método pSnakes podem ser embarcados no próprio equipamento de ultrassom, podendo já processar a imagem obtida, efetuando filtragens e mesmo segmentação de elementos da imagem. No caso do ecocardiograma, a segmentação do ventrículo esquerdo é o objeto de maior interesse. A leitura de suas medidas, em tempo real, traz informações importantes para auxílio ao diagnóstico médico. Este método pode ser aplicado em imagens de natureza cartesiana também, sendo estas imagens primeiramente convertidas para a representação polar, ou mesmo diretamente na representação cartesiana, como no caso de imagens de ultrassom utilizando sonda do tipo linear. Além das imagens de ultrassom, outras imagens de mesma natureza, como as oriundas de sonar ou radar também são naturalmente representadas em coordenadas polares.

Em imagens de ecocardiograma, os resultados nas imagens analisadas são adequados, em relação à segmentação manual do médico especialista. Os resultados obtidos para o nível de significância desejado $(\mathrm{p}<0,05)$ são adequados, mostrando que o pSnakes pode ser utilizado para o cálculo de fração de ejeção em imagens de ecocardiograma em eixo curto, assim como os demais MCA avaliados, exceto $\mathrm{SRD}$. Vale lembrar que a análise de correlação em relação a ECO1 apontou o pSnakes como melhor método. A segmentação de imagens do ventrículo em ecocardiogramas também é adequada, sugerindo que o método pSnakes pode ser utilizado com sucesso na segmentação destas imagens.

Como trabalhos futuros sugerem-se um estudo sobre a inicialização automática do pSnakes, além da aplicação em imagens de ecocardiograma em eixo longo (quatro cavidades). A avaliação da complexidade dos algoritmos utilizados neste trabalho, em especial a do pSnakes deve ser efetuada. Uma avaliação quantitativa da segmentação dos métodos, comparando-se com o pSnakes para imagens de ecocardiograma, baseada em medidas de erro de segmentação, deverá ser feita, além da influência do ruído em imagens sintéticas. A comparação com outros métodos como o GVF e VFC deve também ser realizada. A aplicação do método pSnakes em outras imagens médicas como tomografia computadorizada de pulmões, ressonância magnética cardíaca, entre outras, também deve ser investigada.

\section{Agradecimentos}

Ao Laboratório de Sistemas de Computação - LESC do Departamento de Engenharia de Teleinformática e ao Hospital Universitário Walter Cantídio, ambos da Universidade Federal do Ceará. Ao Laboratório de Ensaios Mecânicos - LEM do Instituto de Educação, 
Ciência e Tecnologia do Ceará. À clínica Prontocárdio em Fortaleza, em especial à Dra. Tereza Cristina P. Diógenes. A Jéssyca Almeida Bessa pelo apoio fundamental na obtenção dos dados experimentais.

\section{Referências}

Alexandria AR, Cortez PC, Felix JHS, Rebouças Filho PP, Cavalcante TS, Silva Júnior JAC, Abreu JS, inventores; Instituto Federal de Educação, Ciência e Tecnologia do Ceará - IFCE. Método de contornos ativos psnakes. MU8903119-9. INPI, Brasil, 2009.

Alexandria AR, Cortez PC, Felix JHS, Cavalcante TS, Rebouças Filho PP, Silva Júnior JAC, Abreu JS. Hilbertian energy: a method for external energy calculation on radial active contours. In: Leta FR, Conci A, editors. Systems, Signals and Image Processing. IWSSIP 2010: Proceedings of the $17^{\text {th }}$ International Conference on Systems, Signals and Image Processing; 2010 Jun 17-19; Rio de Janeiro, Brasil. Rio de Janeiro; 2010. p. 81-4.

Arikidis NS, Skiadopoulos S, Karahaliou A, Likaki E, Panayiotakis G, Costaridou L. B-spline active rays segmentation of microcalcifications in mammography. Medical Physics. 2008; 35(11):5161-71. PMid:19070250. http://dx.doi.org/10.1118/1.2991286

Bednorz W. Advances in Greedy Algorithms. Vienna: INTECH Education and Publishing; 2008.

Bouhours A. Segmentação do ventrículo esquerdo em ecocardiograma usando contornos ativos (snake) [dissertação]. Fortaleza: Universidade Federal do Ceará; 2006. 98p.

Buda AJ, Delp EJ, Meyer CR, Jenkins JM, Smith DN, Bookstein FL, Pitt B. Automatic computer processing of digital 2-dimensional echocardiograms. The American Journal of Cardiology. 1983; 52(3):384-9. http://dx.doi. org/10.1016/0002-9149(83)90144-3

Caiani EG, Lang RM, Korcarz CE, De-Cara JM, Weinert L, Collins KA, Spencer K, Cerutti S, Moravi V. Echocardiographic evaluation of left ventricular wall motion using still-frame parametric imaging. IEEE Computers in Cardiology. 2001; 1(28):89-92.

Chen Y, Huang T, Rui Y. Optimal radial contour tracking by dynamic programming. In: Image Processing. 2001: Proceedings of the IEEE International Conference on Image Processing; 2001 Out 7-10; Thessaloniki, Greece. Thessaloniki; 2001. p. 626.

Cohen LD. On active contour models and balloons. Computer Vision Graphics and Image Processing: Image Understanding. 1991; 53(2):211-8.

Cootes TF, Edwards G, Taylor CJ. Comparing active shape models with active appearance models. In: Pridmore TP, Elliman D, editors. British Machine Vision. BMVC 1999: Proceedings of the British Machine Vision Conference; 1999 Sept 13-16; Nottingham, United Kingdom. Nottingham: BMVA Press; 1999. p. 173-82.

Costa Filho CFF, Moura LA, Costa MGF. Revisão sobre métodos de segmentação $2 \mathrm{D}$ do ventrículo esquerdo. Revista Brasileira de Engenharia Biomédica. 1999; 15(3):141-58.
Dagher I, Tom KE. WaterBalloons: A hybrid watershed Balloon Snake segmentation. Image and Vision Computing. 2008; 26(7):905-12. http://dx.doi.org/10.1016/j. imavis.2007.10.010

Demaine E, Leiserson, C. Introduction to algorithms. Cambridge: MIT Press; 2001.

Denzler J, Niemann H. Active rays: A new approach to contour tracking. International Journal of Computing and Information Technology. 1996; 4:9-16.

Denzler J, Niemann H. Active rays: Polar-transformed active contours for real-time contour tracking. Real-Time Imaging. 1999; 5(3):203-13. http://dx.doi.org/10.1006/ rtim.1997.0116

Ebadollahi S, Shih-Fu C, Wu H. Echocardiogram videos: summarization, temporal segmentation and browsing. In: Image Processing. ICIP 2002: Proceedings 2002 International Conference on Image Processing; 2002 Sept 22-25, New York, USA. IEEE Signal Processing Society; 2002. p. I613-6. http://dx.doi.org/10.1109/ICIP.2002.1038098

Gemignani V, Faita F, Ghiadoni L, Poggianti E, Demi M. A system for real-time measurement of the brachial artery diameter in B-mode ultrasound images. IEEE Transactions on Medical Imaging. 2007; 26(3):393-404. PMid:17354644. http://dx.doi.org/10.1109/TMI.2006.891477

Gemignani V, Paterni M, Benassi A, Demi M. Real time contour tracking with a new edge detector. Real-Time Imaging. 2004; 10(2):103-16. http://dx.doi.org/10.1016/j. rti.2004.02.005

Hodneland E, Tai X-C, Gerdes H-H. Four-color theorem and level set methods for watershed segmentation. International Journal on Computer Vision. 2009; 82(3):264-83. http://dx.doi.org/10.1007/s11263-008-0199-4

Hunter IA, Soraghan JJ, Mcdonagh T. Fully automatic left ventricular boundary extraction in echocardiographic images. In: Computers in Cardiology: Proceedings Computers in Cardiology; 1995 Sept 10-13; Vienna; 1995. p. 741-4.

Johansson M. The Hilbert transform [dissertation]. Växjö: Växjö University; 1999. 33 p.

Kass M, Witkin A, Terzopoulos D. Snakes: active contours models. International Journal on Computer Vision. 1987; 1(4):321-31. http://dx.doi.org/10.1007/ BF00133570

Liang J, Ding G, Wu Y. Segmentation of the left ventricle from cardiac MR images based on radial GVF snake, In: Peng Y, Zhang Y, editors. BMEI 2008: Proceedings of the First International Conference in BioMedical Engineering and Informatics; 2008 May 27-30; Sanya, Hainan, China. IEEE Computer Society; 2008. p. 238-42.

Markman Filho B, Almeida MC, Markman M, Chaves A, Moretti MA, Ramires JAF, César LA. Estratificando o risco na angina instável com a ecocardiografia sob estresse com dobutamina. Arquivos Brasileiros de Cardiologia. 2006; 86(3):259-64.

Marques JAL, Cortez PC, Madeiro JPV. Detecção de alterações da frequência cardíaca fetal e do tônus uterino materno em exames cardiotocográficos utilizando 
transformada de Hilbert. Em: CBEB 2008: Anais do $21^{\circ}$ Congresso Brasileiro de Engenharia Biomédica; 2008 Nov 16-20, Salvador, Bahia. SBEB; 2008. p. 1727-30.

Nixon M, Aguado A. Feature Extraction \& Image Processing. 2th ed. Academic Press: London; 2008.

Osher S, Paragios N. Geometric level set methods in imaging, vision, and graphics. New York: Springer-Verlag; 2003.

Otto CM. Textbook of Clinical Echocardiography. 3th ed. Philadelphia: Elsevier; 2004.

Poularikas AD. The Transforms and applications handbook. 2th ed. Boca Raton: CRC Press; 2000. http://dx.doi.org/10.1201/9781420036756

Sarkar S, Mukherjee K, Ray A. Generalization of Hilbert transform for symbolic analysis of noisy signals. Signal Processing. 2009; 89(6):1245-51. http://dx.doi.org/10.1016/j. sigpro.2008.12.009

Silva JS, Santos BS, Silva A, Madeira J. Modelos deformáveis na segmentação de imagens médicas: uma introdução. Revista do DETUA. 2004; 4(3):360-7.
Sonka M, Hlavac V, Boyle R. Image processing, analysis and machine vision. 3th ed. CL-Engineering; 2007.

Staib LH, Duncan JS. Boundary finding with parametrically deformable models. IEEE Transactions on Pattern Analysis and Machine Intelligence. 1992; 14(11):1061-75. http://dx.doi.org/10.1109/34.166621

Terzopoulos D, Metaxas D. Dynamic 3D models with local and global deformations: deformable superquadrics. IEEE Transactions on Pattern Analysis and Machine Intelligence. 1991; 13(7):703-14. http://dx.doi. org/10.1109/34.85659

Madeiro JPV, Cortez, PC Marques JAL. Nova abordagem de detecção de QRS em ECG através da combinação das transformadas de Wavelet e Hilbert. Em: CBEB 2008: Anais do $21^{\circ}$ Congresso Brasileiro de Engenharia Biomédica; 2008 Nov 16-20, Salvador, Bahia. SBEB; 2008. p. 1731-4.

Webster JG. Medical instrumentation application designer. 3th ed. John Wiley and Sons Inc; 1998.

\section{Autores \\ Auzuir Ripardo de Alexandria \\ Departamento da Indústria, Grupo de Simulação Computacional - G5IMCO, \\ Instituto Federal de Educação, Ciência e Tecnologia do Ceará - IFCE, \\ Av. Treze de Maio, 2081, Benfica, CEP 60040-531, Fortaleza, CE, Brasil}

\section{Paulo César Cortez}

Departamento de Engenharia de Teleinformática, Laboratório de Engenharia de Sistemas de Computação, Universidade Federal do Ceará - UFC, Av. da Universidade, 2853, Benfica, CEP 60020-181, Fortaleza, CE, Brasil

\section{John Hebert da Silva Felix}

Curso de Engenharia de Energias, Universidade da Integração Internacional da Lusofonia Afro-Brasileira - UNILAB, Campus da Liberdade, Av. da Abolição, 3, Centro, CEP 62790-000, Redenção, CE, Brasil

\section{José Sebastião de Abreu}

Hospital Universitário Walter Cantídio, Universidade Federal do Ceará - UFC

Av. da Universidade, 2853, Benfica, CEP 60020-181, Fortaleza, CE, Brasil 